
\title{
R Reserach S Suare \\ The Effect of Schistosomiasis and Soil-Transmitted Helminths on Expressive Language Skills Among isiZulu Speaking Preschool Children
}

Xolisile Innocentia Mazibuko ( $\sim$ mazxoli@gmail.com )

University of Kwazulu-Natal https://orcid.org/0000-0002-0523-2391

Moses Chimbari

University of KwaZulu-Natal College of Health Sciences

\section{Research article}

Keywords: Schistosomiasis, soil-transmitted helminths, expressive language, preschool, isiZulu, South Africa

Posted Date: December 22nd, 2020

DOl: https://doi.org/10.21203/rs.3.rs-132289/v1

License: (c) (i) This work is licensed under a Creative Commons Attribution 4.0 International License.

Read Full License 


\section{Abstract}

Background: Schistosomiasis and soil transmitted helminths (STH) have been associated with compromised child development. We determined the effect of schistosomiasis and STH on expressive language skills among isiZulu speaking preschool children focusing on the variables: age, gender, school and stunting.

Methods: We compared, qualitatively and quantitatively the performance of a cohort of infected and noninfected children using a 2 phased approach. In phase 1 infected children were treated with praziquantel and matched with non-infected children and both groups were tested for expressive language performance. In phase 2 both groups of children were re-tested for expressive language skills using a similar but modified test. The participants were 106 preschool children between the age of 4 and 6 years, 11 months. The Developmental Language Test was adapted as a linguistically and culturally appropriate tool for assessing isiZulu expressive language skills.

Results: The overall performance of the children in phases 1 and 2 were statistically similar. There was significant Pearson's correlation of expressive language skills to age $(0.002, \mathrm{P}<0.01)$, schistosomiasis i.e. vocabulary $1(0.024, \mathrm{P}<0.05)$ and narrative skills $(0.001, \mathrm{P}<0.01)$ and soil-transmitted helminths i.e. vocabulary $1(0.006, \mathrm{P}<0.05)$, colours $(0.029, \mathrm{P}<0.05)$ and narrative skills $(0.001, \mathrm{P}<0.01)$ in phase 2 with small to high Cohen's $d$ effect size for various language subtests.

Conclusion: We concluded that even mild schistosomiasis and STH may compromise the performance of preschool children on expressive language. However poor ability in following instructions may have contributed to general poor performance across the two groups tested. Diet, school effect and stunting did not influence the performance of the children on expressive language.

\section{Background}

Schistosomiasis, also known as Bilharzia, is a chronic debilitating disease, whose public health importance is second to malaria as the most common parasitic condition in Africa (National Institute of Communicable Diseases (NICD), 2018). Two main species of schistosomes infect human beings in Africa - Schistosoma haematobium and Schistosoma mansoni with each species determined by presence of a suitable snail host and its distribution. About 4 million people, mostly children, are infected with schistosomiasis in South Africa (NICD, 2018). Schistosomiasis is transmitted to humans through contact with contaminated water during bathing, swimming, fishing and domestic chores. Thus, the disease is more prevalent in coastal and rural areas of South Africa where there is no running or tap water (Global Atlas of Helminth Infections, 2017). Incidentally, the prevalence of schistosomiasis overlaps with that of soil-transmitted helminths (Global Atlas of Helminths Infections, 2017). The prevalence of soiltransmitted helminths (STH) in KwaZulu Natal is estimated at $38.8 \%$ in school aged children (Molvick et al., 2017). 
Parasitic worms affect human nutrition and growth through a number of routes depending on the species, the mixture of species, the duration of infection and number of worms (Munŏz-Antoli, Parỏn, Pérez, Toledo, \& Estenban, 2017). For instance, intestinal worms feed on the contents of the host's gut and tissues including blood and serum resulting in loss of iron and protein. They can cause maldigestion or malabsorption of the nutrients or induce inflammatory responses that may affect appetite and food intake thereby modifying metabolism and storage of key nutrients. Additionally, contingent responses to infection such as fever and hypertrophy of the muscles also affect behaviour, concentration levels and changes anthropometric status (Munŏz-Antoli, Parỏn, Pérez, Toledo, \& Estenban, 2017). The effect of schistosomiasis and STH on cognitive function could occur as a result of one or a combination of symptoms associated with parasitic infection including iron deficiency anaemia. Since early childhood is a period of rapid growth and development of all organ sytems, particularly the brain, early life iron deficiency results in abnormal neuronal structure. Hence, schistosomiasis has been associated with negative effects on school attendance, scholastic achievement, learning and memory (Ezeamama, Bustinduy, Nkwata, Martinez, Pabalan, Boivin \& King, 2018).

Studies investigating effects of STH infections (Stoltzfus, Kvalsvig, Chwaya, Montressor, Albonico et al., 2001) as well as malaria and anaemia(Dellis, 2010) on neurological development have reported delayed motor and speech development. Neurological consequences are reported even in non-anaemic deficiencies where the potential for learning is reduced due to poor recognition memory and executive functions of infected learners (Luwoski, Koss, Burden, Jonides, Nelson et al., 2010). Early treatment of children with parasitic infections could potentially mitigate the memory and learning deficits in preschool children (Ezeamama, et al., 2018). There is also empirical evidence indicating cognitive and educational benefits of STH treatment (Pabalan, Singian, Tabangay et al., 2018).

Since infection with STH and schistosomiasis are confounding factors to cognitive impairment and developmental delays in communities with poor water and sanitation, there is a knowledge gap on whether STH and schistosomiasis have the same effect on expressive language skills as much as they may have on other cognitive skills. Our study aimed at determining the effect of schistosomiasis and STH on expressive language skills among isiZulu speaking pre-schoolers. We determined the profile of expressive language skills of isiZulu speaking preschool children in terms of age, gender, school, stunting and parasitic infection; and compared expressive language skills for children with and without schistosomiasis and STH.

The knowledge we have on isiZulu grammatical pattern is limited, achronological and has not yet produced developmental language norms for receptive and expressive language (Van der Merwe \& Le Roux, 2014). We know that isiZulu, like Turkish and Finnish, has characteristically agglutinating morphology and in many ways isiZulu is different from English (Keet \& Khumalo, 2016). Data collected from similar Nguni languages such as isiXhosa showed that trilingual and monolingual children had similar lexical development in the languages the children were exposed to (Potgieter, 2016). Expressive language builds up from nouns to verbs, adjectives, adverbs, prepositions, conjunctions and auxiliary verbs (Gonasilan, Bornman \& Harty, 2013). Typical emergent skills for expressive language are memory, 
time-based relations, cause and effect reasoning, social script, sense of self and vocabulary which lead to learning colours, questions and narrative skills can all be achieved as early as 36 months (Phillips, 2008).

Expressive language development is usually described using the standards set in English according to the Brown's stages of syntactic and morphological development where children begin to express their thoughts in single words around 12 months and achieve full sentences and narrative skills by 5 years (Bowen, 1998). By the age of 5 years children are usually enrolled in a preschool where early identification of at-risk children and the provision of appropriate interventions and support can substantially affect future scholastic progress (Wildschut, Moodley \& Aronstam, 2016). Since attendance of grade R has been found to influence literacy development, we decided to determine expressive language skills in children attending a preschool (Daws, Biersteker, Girdwood, Snelling \& Tredaux, 2018).

The main risks associated with expressive language development in English speaking children has been shown to include a variety of factors such as family dynamics, interaction with parents, immediate social environment, organic hazards such as brain injury, persistent otitis media, types of food (Gurger, Vidor, Joly \& Reppold, 2014), a reactive temperament and being male (Harrison \& McLeod, 2010). Hence, we examined the effect of age, gender, school and status of infection with schistosomiasis and STH on expressive language skills in our sample.

\section{Methods}

\section{Design and setting of the study}

Our study area was a small rural town of Ingwavuma, located in northern KwaZulu-Natal province of South Africa, close to the borders of eSwatini (in the North) and Mozambique (in the east). Ingwavuma is under traditional authority and is regarded as the worst poverty-stricken area in KwaZulu-Natal with an estimated annual income of R32 812 (STATSSA 2015, p17). Most people in this area depend heavily on social grants estimated to R13 090 per annum (less than USD1000) as their main source of income, (General household surveys, 2018). The prevalence of Schistosoma haematobium in Ingwavuma is $37.5 \%$ amongst school attending children above 10 years (Kabuyaya, Chimbari, Manyangadze \& Mukaratiwa, 2017) and for young children (1-5 years) it is $2 \%$ for both $S$. haematobium and S. mansoni. The risk factors for schistosomiasis among young children include caregiver's age, type of household head, poor sanitation, access to water source and knowledge about schistosomiasis (Sacolo-Gwebu, Chimbari, \& Kalinda, 2019).

The study was an analytical cohort study which described and compared language skills of non-infected and infected children with schistosomiasis and STH using clinical assessments and observations to obtain data. It was an ancillary study to the Tackling infections to benefit Africa (TIBA SA) project (http://tiba-partnership.org/about/what-is-tiba). Over 700 preschool children were tested for STH and schistosomiasis in the TIBA study between 2017 and 2020. Schistosoma haematobium was diagnosed using the filtration technique on urine samples (Mott, 1983); S. mansoni and STH (Taenia, Ascaris 
Lumbricoides, Trichuris Trichiura) were diagnosed on stool samples using the Kato Katz technique (Katz, Chaves \& Pellegrino (1972).

Purposeful random sampling was used. To be eligible for the study a child had to be of age between 4.0 and 6.11 years; attend an isiZulu medium preschool or ECD in the target area, have no developmental delays, be monolingual isiZulu speaker and pass a hearing screening test. The study participants were striated based on age ( 4 to 6 years), gender (50\% of each gender) and inclusion of infection positive participants at a ratio of 2 negative cases for every 1 positive case. The principal researcher was blinded to both status and nature of infection of the study participants. Children were tested in two phases; phase 1 was testing immediately after parasitology screening and phase 2 was testing at least 12 weeks later and after treatment of children with schistosomiasis and STH with oral Praziquantel. Some children participated in both phases while some participated only in phase 1 and could not be traced for repeat testing. The data for the children who participated in both phases was treated separately because the phases were 3 months apart, affecting the children's level of maturity and the language test was adapted for phase 2 to reduce content bias.

\section{Sample characteristics}

The distribution of children in phase 1 and 2 varied in terms of age, gender and infection. The mean age was 4 years 9 months in phase 1 and 5 years 9 months in phase 2 . The total number of children with schistosomiasis (17.9\%) was less than the number of children with STH (31.1\%) and the infections were not necessarily co-existing. The combined distribution of infections in phases 1 and 2 indicated that the largest number of STH infection was from A. Lumbricoides with 17 cases (16\%). 
Table 1

sample characteristics

\begin{tabular}{|llll|}
\hline Variable & Description & Phase 1 & Phase 2 \\
\hline Gender & Boys & $\mathrm{N}=38$ & $\mathrm{~N}=23$ \\
& Girls & $\mathrm{N}=20$ & $\mathrm{~N}=25$ \\
& Total N & $\mathrm{N}=58$ & $\mathrm{~N}=48$ \\
\hline Age & Minimum & 4.0 & 4.10 \\
& Maximum & 6.11 & 7.03 \\
& Mean & 4.9 & 5.7 \\
Schistosomiasis & Positive $(1)$ & $19(32.75 \%)$ & $13(27 \%)$ \\
\hline STH \& number of infections carried by 1 child & STH Total & $\mathrm{N}=19(32.75 \%)$ & $\mathrm{N}=16(33 \%)$ \\
& 1 & $15.5 \%$ & $7=(14,5 \%)$ \\
& 2 & $12.1 \%$ & $8=(16,6)$ \\
& 3 & $5.2 \%$ & $1=(2.1 \%)$ \\
\hline Time Taken to Test & Mean & 26.97 min & $23.71 \mathrm{~min}$ \\
\hline
\end{tabular}

The 17 participating preschools had pit toilets, at least one rainwater harvest tank within the facility and provided one meal a day for the children. In our observations on all test dates, the meals did not include green leafy vegetables or meat. All the teachers met the minimum required qualification of high school education for ECD and a teaching diploma for Grade R. Although all schools had government supplied grade R books, they lacked in resources such as puzzles, board games, a computer, a television and had no internet access.

\section{Data collection procedures}

Considering the socio-economic profile of the study area, children were provided with a peanut butter sandwich and orange juice before undergoing testing to ensure they had a healthy breakfast and had energy to participate in the tests. Testing started with hearing screening which, included otoscopic examination and tympanometry in order to exclude ear infections and its contribution to poor language scores (Harrison \& McLeod, 2010). A nutritionist determined the participant's anthropometric data by calculating BMl-for-age (weight in kg) and height-for-age (height and arm circumference in $\mathrm{cm}$ ) to determine stunting (World Health Organisation, 2011). The children were classified as having mild (1), moderate (2) or severe (3) levels of stunting. The majority of the participants had adequate nutrition. The prevalence of stunting was $26 \%$ and the results showed no significant correlation of the test scores to stunting in both phases of the study. 
All children were monolingual, speaking isiZulu at home and at school. The Developmental Language Test (Kvalsvig, Govender \& Taylor, 2009), a non- standardised test developed for a research project, was adapted for this study following a pilot study and observations by research assistants. From these observations the test's vocabulary was adjusted to include local dialect (Northern KwaZulu-Natal coast) and the sequence of test items was formatted into 5 sections for easy scoring and interpretation (Table 2). New test illustrations were developed using pictures taken in Ingwavuma with local community members for improved clarity and to accommodate the adjusted format of the test (see appendix A for test illustrations and B for test form).

Table 2

Adapted Developmental Language Test Score form (Kvalsvig, Govender, \& Taylor, 2009)

\begin{tabular}{|c|c|c|c|c|c|c|c|}
\hline \multirow[t]{2}{*}{ Subtest } & \multirow[t]{2}{*}{ Task } & \multirow[t]{2}{*}{ Notes } & \multicolumn{5}{|c|}{ Sum Score } \\
\hline & & & 4 & 3 & 2 & 1 & 0 \\
\hline \multirow[t]{2}{*}{ 1.Vocabulary 1(nouns) } & Sing a song & & & & & & \\
\hline & $\begin{array}{l}\text { Identify people \& } \\
\text { names }\end{array}$ & & & & & & \\
\hline \multirow[t]{2}{*}{ 2.Colours } & Recognition & & & & & & \\
\hline & Naming & & & & & & \\
\hline \multirow[t]{4}{*}{ 3.Answering WH-Questions } & Who & & & & & & \\
\hline & What & & & & & & \\
\hline & Where & & & & & & \\
\hline & Why & & & & & & \\
\hline \multirow{5}{*}{$\begin{array}{l}\text { 4. Vocabulary (Verbs and Object } \\
\text { function) }\end{array}$} & Counting1-15 & & & & & & \\
\hline & Object naming & & & & & & \\
\hline & Object function & & & & & & \\
\hline & Meanings & & & & & & \\
\hline & Categorisation & & & & & & \\
\hline \multirow[t]{2}{*}{ 5.Narrative skills } & Inferencing & & & & & & \\
\hline & Story retelling & & & & & & \\
\hline TOTAL & WHOLE TEST & & & & & & \\
\hline
\end{tabular}

The adapted Developmental Language Test showed a sensitivity of $89.7 \%$ in phase 1 and $81.3 \%$ in phase 2 reflecting that children who tested positively were true positives while specificity was 10.3 and $18.8 \%$ respectively. The Cronbach's Alpha was determined to be $0.869(S D=5.1)$ in phase 1 and $0.813(S D=7.7)$ 
in phase 2 demonstrating adequate internal consistency and suggesting that all items measured the same construct.

The test was administered to one child at a time by the principal researcher who is a speech-language therapist and an isiZulu speaker, familiar with dialect and culture of the area. Scoring points varied from 0 to 4 points per target, depending on the extent of the answer as described in the test form (APPENDIX B). Scoring was immediate and automated via an adapted kobo collect app, an open source platform used for collecting and analysing data (Palla, LeBel \& Chavernac, 2016).

\section{Data analysis}

Quantitative data analysis for both phases comprised of descriptive frequency analysis, Independent Samples t Tests, ANOVA and Bivariate correlations on the SPSS 25 program. Post hoc tests were conducted using Bonferroni corrections to measure the specific contribution of variables such as age, gender, school and infection on language categories and time taken to complete the test. The overall error rate was controlled by the use of adjusted significance levels $(a=0.05)$. Information processing model for cognitive skills (Malmberg, Raaijmakers \& Shiffrin, 2019) and Vygotsky's sociocultural theory for development and learning guided data analysis and interpretation of scores (Vygotsky, 1978/ 1995).

\section{Results}

The results showed no significant differences in language scores due to STH or schistosomiasis infections in the pre-treatment Phase 1. However, in Phase 2, there was correlation of schistosomiasis to two language subtests i.e. vocabulary $1(0.024, \mathrm{P}<0.05)$ and narrative skills $(0.001, \mathrm{P}<0.01)$. There was medium correlation effect size for correlation of schistosomiasis to vocabulary 1 (Cohen's $d=-0.67$ ), a medium effect size for colours (Cohen's $\mathrm{d}=\mathrm{-0.53}$ ) and a high effect size for correlation of schistosomiasis to narrative skills subtest (Cohen's $\mathrm{d}=-\mathbf{0 . 9 9}$ ). The correlation was negative for all three subtests indicating that schistosomiasis may have contributed to the reduction of the mean. There was also correlation of expressive language scores to STH for three subtests i.e. vocabulary $1(0.006, \mathrm{P}<0.05)$, colours $(0.029, \mathrm{P}$ $<0.05)$ and narrative skills $(0.001, \mathrm{P}<0.01)$. The correlation effect size of STH on vocabulary 1 was negative (Cohen's $d=-1.84$ ), while for vocabulary 2 it was small and positive (Cohen's $d=0.28$ ). The effect size for colours (Cohen's $d=-1.53$ ) and narratives (Cohen's $d=-2.16$ ) was negative. Statistically, there was no correlation between the test scores and the school factor.

There were significant differences in performance by age for four subtests in phase 1 (excluding $\mathrm{WH}$ Questions) and three subtests in phase 2 (excluding narrative skills and vocabulary 2) were established $(P=0.028 ; P<0.05)$. The analysis of the correlation effect size indicated that the effect was mainly negative, with a moderate effect size for vocabulary 1 (Cohen's $d=-0.84$ ); and a small effect size for vocabulary 2 (Cohen's $d=-0.21$ ).

Boys performed better than girls in vocabulary subtests 1 and 2 respectively $(0.022, \mathrm{P}<0.05)$ in phase 1 and in phase $2(0.023, \mathrm{P}<0.05)$. Similar observations were made in the colours, WH-questions and 
narratives tests. The correlation effect size for gender was below minimal (Cohen's $d=0.024$ for boys and 0.022 for boys). The mean time taken to complete the test (TTT) was 26.97 minutes in phase 1 and 23.71 minutes in phase 2 and positively correlated to age but not gender $(0.002, P<0.01)$ for phase 1 and phase $2(0.28, \mathrm{P}<0.05)$.

Analysis of the total cohort performance on each subtest indicated the following trends which are relevant for clinical language norms: In the Vocabulary 1 subtest which, included tasks to recall character names, pointing to characters and objects on picture A, few children achieved above the mean (total score was 12) on the task. For the second subtest which required identification and naming of colours, the majority of children did not know all their primary colour names and achieved the mean score of 3 in both phases. The third subtest required answering $\mathrm{WH}$ - questions in isiZulu where the majority of the children could not obtain the full score in this section mainly because they could not answer the Why question. The Vocabulary 2 subtest various aspects of vocabulary including verbs, numbers and categorisation of items. There was minimal variability in the children's performance (Table 3 ).

Table 3

Expressive language profile comparison between Phase $1 \& 2$

\begin{tabular}{|llllllll|}
\hline Subtest & \multicolumn{2}{l}{ Mean Raw score } & S.D. & & Sig. & \multicolumn{2}{l|}{ Sig (2-tailed) } \\
\hline & Phase1 & Phase 2 & Phase1 & Phase2 & & Phase 1 & Phase 2 \\
\hline 1. Vocab1 & 8.2 & 9.0 & 2.21 & 2.23 & .093 & .098 & .105 \\
\hline 2. Colours & 3.1 & 3.9 & 1.8 & 1.3 & .002 & .012 & .009 \\
\hline 3. Wh- Questions & 2.4 & 3.5 & 0.84 & 0.89 & .539 & .000 & .000 \\
\hline 4. Vocab 2 & 13.1 & 20.3 & 3.2 & 2.3 & .015 & .000 & .000 \\
\hline 5. Narratives & 8.2 & 6.9 & 2.5 & 2.2 & .411 & .005 & .004 \\
\hline Test Total Sum & 35.2 & 43.8 & $\mathbf{8 . 3}$ & 7.7 & .349 & .000 & .000 \\
\hline
\end{tabular}

Overall, the results showed similar trends in both phases as there was no significant difference between the means of different language subtests in both phases for the cohorts. The age specific results indicate that age contributed to variation of the total scores with the older children performing better in language subtests (Table 4). 
Table 4

Mean Raw score per subtest and age band

\begin{tabular}{|llllllll|}
\hline $\begin{array}{l}\text { Age } \\
\text { band }\end{array}$ & $\mathbf{N}$ & $\begin{array}{l}\text { Mean Voc } \\
\mathbf{1}\end{array}$ & $\begin{array}{l}\text { Mean } \\
\text { Colours }\end{array}$ & $\begin{array}{l}\text { Mean } \\
\text { Q\&A }\end{array}$ & $\begin{array}{l}\text { Mean Voc } \\
\mathbf{2}\end{array}$ & $\begin{array}{l}\text { Mean } \\
\text { Narratives }\end{array}$ & $\begin{array}{l}\text { Mean } \\
\text { Total }\end{array}$ \\
\hline $4.0-4.5$ & 8 & 8 & 1.8 & 2.6 & 12 & 7.5 & 32 \\
\hline $\begin{array}{l}4.6- \\
4.11\end{array}$ & 6 & 7.6 & 1.5 & 2.3 & 12.5 & 6.6 & 30.2 \\
\hline $5.0-5.5$ & 20 & 8.25 & 3.25 & 2.4 & 12.45 & 8.7 & 34 \\
\hline $\begin{array}{l}5.6- \\
5.11\end{array}$ & 12 & 9.25 & 3.17 & 2.25 & 13.3 & 8.4 & 36.3 \\
\hline $6.0-6.5$ & 4 & 9.75 & 5 & 3 & 16.7 & 10.5 & 44.7 \\
\hline $\begin{array}{l}6.6- \\
6.11\end{array}$ & 7 & 9.4 & 4.7 & 3 & 16.3 & 11 & 43.3 \\
\hline
\end{tabular}

Comparing the five subtest's medians to the 25th and the 75th percentiles, the medians were equidistant from the quartiles indicating normal distribution of data. There was minimal variability in the children's performance considering the percentile ranks and narrow interquartile range (Table 5).

Table 5

Percentiles

\begin{tabular}{|llll|}
\hline Subtest & 25th P & 50th P & 75th P \\
\hline Vocab 1 & 47 & 51 & 52 \\
\hline Colours & 43 & 46 & 51 \\
\hline Wh Questions & 45 & 48.5 & 51 \\
\hline Vocab 2 & 49 & 52 & 52 \\
\hline Narratives & 39.5 & 45 & 46 \\
\hline
\end{tabular}

\section{Discussion}

The children came from similar backgrounds and comparable schooling contexts hence, no significant differences were noted between the schools and all differentiating variables analysed. The comparison of language scores from the pre-treatment and post treatment phases indicate that the means were higher in phase two. Since we had controlled for content bias by adapting the language test and changing the test pictures in phase 2, the results imply that the older the children the higher the scores in all test areas. The gender comparison in this study shows that boys performed better in vocabulary subtests. This is an unusual finding as many studies report girls as having stronger expressive or oral language skills and boys at a higher risk of speech and language impairment (Prathanee, Thinkhamrop, \& Dechongkit, 2007; Harrison \& McLeod, 2010; Tsai et al., 2017). Since gender related differences in language acquisition 
have been attributed to cortical sex differences, thought to be acquired or enlarged through different stages of development and attributed to different cognitive strategies between sexes, we recommend further investigation into the factors that may have contributed to the trends in our study, considering its rural setting and specific to isiZulu language.

There was a positive effect of age on TTT, implying that with the increasing children's age, the speed to complete the test also increased. We regard the effect size minimal as it fell 1 point below the recommended 0.25 (Geata \& Brydges, 2020). This was an expected finding and in line with the information processing model and cognitive theories of language development such as Luria's neurological learning theory (Clark, 2004). We did not find significant correlation between children with and without schistosomiasis and STH in TTT. Uniform processing time was observed for candidates with and without schistosomiasis in learning and memory tests in similar studies (Ezeamama et al., 2018). Similarly, when STH was associated with learning, memory and intelligence, there was no significant correlation of testing time with infection (Pabalan et al., 2018).

The results showed a negative effect of infection to language subtests where the correlation was significant in the post treatment phase 2 suggesting that children with history of parasitic infections performed lower than their peers and thus explaining the reduction of the mean of the relevant tests. This negative effect size was medium for vocabulary and colours subtests but high for narratives skills. Narrative tasks required the ability to follow commands and retell a story in sequence, skills that require application of short term (ability to retain information for a short time) and working memory (the ability to maintain activated knowledge in the focus of attention) (Engle, Tuholski, Laughlin \& Conway, 1999). There is evidence of a relationship between linguistic knowledge and verbal working memory (VWM) in that linguistic knowledge impact on vWM (Kowialiewski \& Majerus, 2019) and that vWM depends on long term representations and processes that are involved in speech production (Acheson, Hamidi, Binder \& Postle, 2011). Therefore, we are intrigued by our results which imply a causal relationship between schistosomiasis and weaker narrative skills in the light of a known relationship between parasitic infection and memory deficits. Furthermore, the findings reveal the possibility of long term deficits in executive functions and memory after the parasitic worm infection and iron deficiencies have resolved (Luwoski et al., 2010).

The prevalence of STH and Schistosomiasis in our study was similar to that of other studies conducted in KwaZulu-Natal (Zulu, Ekjetland, Gundersen, \& Taylor, 2020) but less than the prevalence in other lowand middle-income countries (Welsch, Hossain, Ghogamu et.al., 2019). The intensity of infection with both STH and schistosomiasis was mild and the majority of the participants were found to have adequate nourishment. We regard this as an important finding as the participants in this study area were from a typical low socio-economic rural context and their school diet was not particularly rich in recommended nutrients such as omega 3 polyunsaturated fatty acids (Spencer, Karosi, Laye, Shukitt-Hale \& Barrientos, 2017). Further investigation of the children's home nutrition and other possible contributing contextual factors would have provided more conclusive results. Thus, one may conclude that the consequences of moderate to severe infection with Schistosomiasis and STH such as malnutrition do 
not apply to this study population (Gwetu, Taylor, Chhagan, Kauchali \& Craib, 2019). This finding has positive implications for the accuracy of the profile of language skills reflected by the findings. We recommend involvement of parents through a direct questionnare and to obtain more data on the socioeconomic factors impacting on the children's health related quality of life and specific information on language acquisition.

\section{Conclusion}

Schistosomiasis mainly negatively impacted on language scores, with a medium effect for vocabulary and colours and a high effect for the narrative subtest. Our findings illustrate the contribution of verbal working memory on expressive language abilities. Our findings also showed correlations of expressive language performance to age as well as correlations of vocabulary subtests to gender (boys), STH and schistosomiasis. Socio-economic factors such as diet, preschool and stunting did not contribute to the scores. The adapted Developmental Language Test offered valuable information towards our understanding of the profile of abilities of isiZulu speaking preschool children. The findings of this study are relevant for all professionals involved in the education and rehabilitation of preschool children with infectious diseases and emphasise the need for treatment of mild STH and Schistosomiasis.

\section{Declarations}

\section{Ethics Approval:}

The study was approved by the University of KwaZulu-Natal BRICS committee (Reference BE 252/19) and parental consent forms were signed for all participants.

\section{Consent for publication:}

All consents were obtained. The NIRH had no interest in writing or submitting the article for publication.

\section{Availability of supporting data:}

Data sets used and analysed during the current study are available from the corresponding author on reasonable request.

\section{Competing interests:}

The authors declare that they have no competing interests.

\section{Funding:}

The TIBA research study was commissioned by the National Institute for Health Research (NIHR) Global Health Research programme (16/136/33) using UK aid from the UK Government. The recipient was Prof M. Chimbari as a principal Investigator. DrX. Mazibuko did not receive any funding from any 
organisation for this follow up study on expressive language. The views expressed in this publication are those of the author(s) and not necessarily those of the NIHR or the Department of Health and Social Care.

\section{Author's Contributions:}

Author 1 conducted the investigations, adapted the language test, contributed to the design of the methodology and wrote the original draft of this article.

Author 2 was responsible for conceptualization of the study, was involved in the writing of this article through reviews and editing and sought the funding for the project.

\section{Acknowledgements:}

The University of KwaZulu-Natal also supported the study through the College of Health Sciences Scholarship. The authors wish to thank the community research assistants for assisting with access to participants and data collection.

\section{Author's information}

Author 1:

Name: Xolisile I. Mazibuko PhD.

ORCl: https://orcid.org/0000-0002-0523-2391

Type of affiliation: Research Fellow

School of Nursing and Public Health

College of Health Sciences

University of KwaZulu-Natal

Phone: +27357722999 (office)

+27729015161 (mobile)

Email:mazxoli@gmail.com;

mazibukox2@ukzn.ac.za

Author 2:

Name: Moses Chimbari (PHD, Prof.)

ORCl: https://orcid.org/0000-0001-8109-8801 
Type of affiliation: Research Professor - Public Health

School of Nursing and Public Health

College of Health Sciences

University of KwaZulu-Natal

Phone: +27312604833 (office)

+27605086969 (mobile)

Email: Chimbari@ukzn.ac.za

\section{ADDRESS OF AFFILIATING INSTITUTION}

College of Health Sciences

School of Public Health, George Campbell Building

Howard College Campus

Durban 4001

South Africa

\section{References}

1. Acheson D, Hamidi M, Binder J, Postle B. A common neural substrate for language production and verbal working memory. J Cog Neurosci. 2011;23(6):1358-67.

2. Bowen C. (1998). Brown's Stages of Syntactic and Morphological Development. \&view=article\&id=33 on 2020.07.19.

3. Clark E. How language acquisition builds on cognitive development. Trends in Cognitive Science. 2004;8(10):472-8.

4. Daws A, Biersteker L, Girdwood E, Snellng M, Tredaux C. Early learning assessment innovation in South Africa: a locally appropriate monitoring tool. Childhood Education. 2018;94(1):12-6.

5. Dellis A. (2010). The behaviour and development of infants with iron deficiency anaemia: systematic observation of 9-month Pemban caregiver infant dyads [PhD Thesis]. Durban: University of KwaZulu Natal.

6. Engle R, Tuholski S, Laughlin J, Conway a. Working memory, short term memory and General Fluid intelligence: A latent-Variable Approach. J Exp Psychol. 1999;128(3):309-31.

7. Ezeamama A, Bustinduy A, Nkwata A, Martinez L, Pabalan N, Boivin M, King C. Cognitive deficits and educational loss in children with schistosome infection-A systematic review and meta-analysis. PloS 
Neglected Tropical Diseases. 2018;21(2):ecollection. https://doi.org/10.1371/journal.pntd.0005524.

8. Geata L, Brydges C. An examination of effect sizes and statistical power in speech, language and hearing research. JSLHR. 2020;63(5):1572-80.

9. Global Atlas of Helminths Infections. (2017, March 29). Distribution of soil transmitted helminths survey data and average district level prevalence. Global Atlas of Helminth Infections:www.thiswormyworld.org.

10. Gurger L, Vidor D, Joly M, Reppold C. Risk factors for proper oral language development in children: a systematic literature review. CODAS. 2014;26(5):350-6.

11. Gwetu T, Taylor M, Chhagan M, Craib M, Kauchali S. Health and education achievement of school aged children:The impact of aneamia and iron status on learning. Journal of Interdisciplinary Health Science. 2019;24:e. https://doi.org/10.4102/hsag.v24i0.1101.

12. Harrison L, \& S.McLeod. Risk and protective factors associated with speech and language imprairment in a nationally representative sample of 4-5 year old children. J sp Lang Hear Res. 2010;53(2):508-29.

13. Katz N, Chaves A, Pellegrino J. A simple device for quantitative stool thick-smear technique in Schistosomiasis mansoni. Rev Inst Med Trop Sao Paulo. 1972;14(6):397-400. PMID:4675644.

14. Khoza-Shangase $K$, Mophosho M. LAnguage and culture in speech- language and hearing professions in South Africa: the dangers of a single story. South Afr J Commun Disord. 2018;65(1):a594.

15. Kowialiewski B, Majerus S. (2019). Verbal working memory and linguistic long-term memory: Exploring the lexical cohort effect. Memory \& Cognition, 47, 997-1011. https://link.springer.com/article/10.3758/s13421-019-00898-5.

16. Keet M, Khumalo L. (2016). Grammar rules for the isiZulu complex verb. Southern African Linguistics \& Applied language studies, 35 (2).

17. Kvalsvig J, Govender K, Taylor M. Research on the age validation of NELDS related to the cognitive development of children between 0 and 4 years of ages. Pretoria: UNICEF and the Department of Education; 2009.

18. Lozoff B, Clark K, Jing Y. Dose-response relationships between iron deficiency with or without anemia and infant social-emotional behaviour. J Peadiatr. 2008;152:696-702.

19. Luwoski AF, Koss M, Burden MJ, Jonides J, Nelson C. Iron deficiency in infancy and neurocognitive function at 19 years: evidence of long term-deficits in executive function and recognition memory. Nutr Neurosci. 2010;13:54-70.

20. Malmberg K, Raaijmakers J, Shiffrin R. (2019). 50 years of research sparked by Atkinson \& Shiffrin (1968). Memory \& Cognition, 561-574. https://doi.org/10.3758/513421-019-0089607.

21. Molvik M, Helland E, Zulu S, Kleppa E, Lillebo K, Gundarsen S, Kvalsvig J, Taylor M, Kjetland E, Vennersvald B. Coinfection with schistosoma haematobium and soil- transmitted helminths in rural South Africa. S Afrjsci. 2017;113(3-5):1-6. 
22. National Institute of Communicable Diseases (NICD). (2018, Nov 23). Schistosomiasis FAQ document. Retrieved from National Institiute of Communicable diseases: nicd.ac.za/wpcontent/uploads/2017/03/schistosomiasis.

23. Pabalan N, Singian E, Tabangay L, Jarjanazi H, J.Boivin M, Ezeamama EA, Budke C. Soil-transmitted helminth infection, loss of education and cognitive impairment in school-aged children: A systematic review and meta-analysis. Plos Neglectable Tropical Diseases. 2018;12(1):e. https://doi.org/10.1371/journal.pntd.0005523.

24. Palla F, LeBel S, Chavernac D. New technologies: mobile data collection system implication for wildlife management in central Africa. In: Sist P, Carriere P, Parolin P, P. \& et Forget P, editors. Tropical ecology and society reconciliating conservation and sustainable use of biodiversity. Montpellier: France: Association for topical biology and Conservation (ATBC); 2016.

25. Phillips L. (2008). Handbook of language and literacy development: a roadmap from 0-60 months. Canadian language and literacy research network. Retrieved from: the roadmap.ualberta.ca on 2020.07.19.

26. Prathanee B, Thinkamrop B, Dechongkit S. (2007). Factors associated with specific language impairment and later language development during early life: a literature review. [Published correction appears in Clin. Pediatr. (phila), 46 (1): 22 - 9.

http://doi.org.10/1177/0009922806297153.

27. Potgieter AP. (2016). Lexical and grammatical development in trilingual speakers of isiXhosa, English and Afrikaans. South African Journal of Communication Disorders, 61 (1)..

28. Sacolo-Gwebu H, Chimbari M, Kalinda C. (2019). Prevalence and risk factors of schistosomiasis and soil-transmitted helminthiases among preschool aged children (1-5 years) in rural KwaZulu-Natal, South Africa: a cross-sectional study. Infectious Diseases of Poverty, 8(1). https://doi.org/ 10.1186/s40249-019-0561-5.

29. Spencer S, Karosi A, Laye S, Shukitt-Hale B, Barrientos R. Food for thought: how nutrition impacts cognition and emotion. Npj Science of Food. 2017;1(7):e1038.

30. Stoltzfus R, Kvalsvig J, Chwaya H, Montressor A, Albonico M, Tielsch M, Savioli L.,Pollitt E. Effects of iron supplementation and anthelmintic treatment on motor and language development of preschool children in Zanzibar:double blind, placibo controlled study. BMJ. 2001;323(7326):1389-93.

31. Tsai C, Chen V, Yang Y, Hung T, Hueng ML, K., \& Gossop M. The association between mycoplasma pneumonia infection and speech \& language imparement: a nationwide population based study in Taiwan. Plos One. 2017;12(7):e180402.

32. Van der Merwe A, Le Roux M. (2014). Idiosyncratic sound systems of the South African Bantu languages: research and clinical implications for speech-language pathologists and Audiologists. South African Journal of Communication Disorders, 61 (1)..

33. Vygotsky L. (1978/ 1995). Mind in Society: Development of higher psychological processes. (M. Cole, V. John-Steiner, S. Schriber, \& E. Souberman, Eds.) Cambridge, MA: Havard University Press. 
34. Wildschut Z, Moodley T, Aronstam S. The baseline assessment of grade 1 learners' literacy skills in a socio-economically disadvantaged school setting. South African Journal of Childhood Education. 2016;6(1):a340.

35. World Health Organisation. (2011). "Haemoglobin Concentrations for the Diagnosis of Anaemia and Assessment of Severity." Geneva: World Health organisation. http://www.who.int/vmnis/indicators/haemoglobin/en/.

36. Zulu S, Ekjetland S, Gundersen S, Taylor M. Prevalence and intensity of neglected tropical diseases (Schistosomiasis and soil-transmitted helminths) amongst rural female pupils in Ugu district, KwaZulu-Natal, South Africa. Southern Africa Journal of Infectious Diseases. 2020;35(1):a123.

\section{Supplementary Files}

This is a list of supplementary files associated with this preprint. Click to download.

- AppendixABEdited.docx 